\title{
Intermittent Versus Continuous Low-Energy Diet in Patients With Type 2 Diabetes: Protocol for a Pilot Randomized Controlled Trial
}

Sarah McDiarmid ${ }^{1 *}$, RD, MMath; Michelle Harvie ${ }^{1 *}, \mathrm{RD}, \mathrm{PhD}$; Rhona Johnson ${ }^{2}$, RGN, RSCN; Avni Vyas ${ }^{1}, \mathrm{RD}$, MPhil; Azza Aglan ${ }^{3}$, PhD, ClinPsyD; Jacqui Moran ${ }^{2}$, RD; Helen Ruane ${ }^{1}$, BSc; Amanda Hulme ${ }^{2}$, RGN, BSc; Katharine Sellers ${ }^{1}$, BSc; Basil Issa ${ }^{2 *}$, MD, FRCP

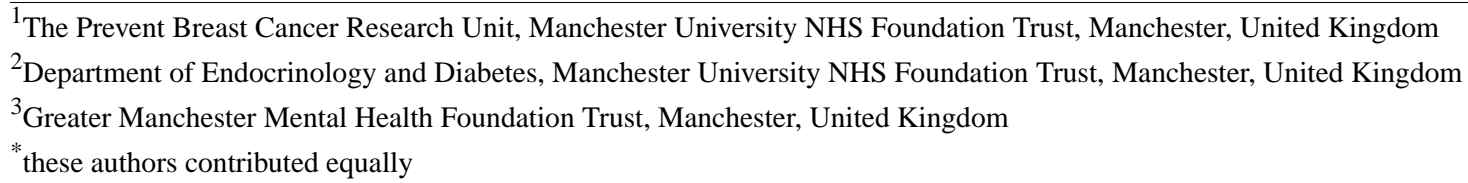

\section{Corresponding Author:}

Sarah McDiarmid, RD, MMath

The Prevent Breast Cancer Research Unit

Manchester University NHS Foundation Trust

Southmoor Road

Wythenshawe Hospital

Manchester, M23 9LT

United Kingdom

Phone: 441612914412

Fax: 441612914421

Email: sarah.mcdiarmid@mft.nhs.uk

\section{Abstract}

Background: Intensive face-to-face weight loss programs using continuous low-energy diets (CLEDs) providing approximately $800 \mathrm{kcal}$ per day (3347 kJ per day) can produce significant weight loss and remission from type 2 diabetes (T2D). Intermittent low-energy diets (ILEDs) and remotely delivered programs could be viable alternatives that may support patient choice and adherence.

Objective: This paper describes the protocol of a pilot randomized controlled trial to test the feasibility and potential efficacy of remotely supported isocaloric ILED and CLED programs among patients with overweight and obesity and T2D.

Methods: A total of 79 participants were recruited from primary care, two National Health Service hospital trusts, and a voluntary T2D research register in the United Kingdom. The participants were randomized to a remotely delivered ILED ( $n=39$ ) or CLED $(n=40)$. The active weight loss phase of CLED involved 8 weeks of Optifast $820 \mathrm{kcal} / 3430 \mathrm{~kJ}$ formula diet, followed by 4 weeks of food reintroduction. The active weight loss phase of ILED ( $\mathrm{n}=39)$ comprised 2 days of Optifast $820 \mathrm{kcal} / 3430 \mathrm{~kJ}$ diet and 5 days of a portion-controlled Mediterranean diet for 28 weeks. Both groups were asked to complete 56 Optifast $820 \mathrm{kcal} / 3430 \mathrm{~kJ}$ days during their active weight loss phase with an equivalent energy deficit. The diets were isocaloric for the remainder of the 12 months. CLED participants were asked to follow a portion-controlled Mediterranean diet 7 days per week. ILED followed 1-2 days per week of a food-based $820 \mathrm{kcal} / 3430 \mathrm{~kJ}$ diet and a portion-controlled Mediterranean diet for 5-6 days per week. Participants received high-frequency (weekly, fortnightly, or monthly depending on the stage of the trial) multidisciplinary remote support from a dietitian, nurse, exercise specialist, and psychologist via telephone or the Oviva smartphone app. The primary outcomes of the study were uptake, weight loss, and changes in glycated hemoglobin at 12 months. An outcome assessment of trial retention was retrospectively added. Secondary outcomes included an assessment of adherence and adverse events. A qualitative evaluation was undertaken via interviews with participants and health care professionals who delivered the intervention.

Results: A total of 79 overweight or obese participants aged 18-75 years and diagnosed with T2D in the last 8 years were recruited to the Manchester Intermittent and Daily Diet Diabetes App Study (MIDDAS). Recruitment began in February 2018, and data collection was completed in February 2020. Data analysis began in June 2020, and the first results are expected to be submitted for publication in 2021.

Conclusions: The outcomes of the MIDDAS study will inform the feasibility of remotely delivered ILED and CLED programs in clinical practice and the requirement for a larger-scale randomized controlled trial. 
Trial Registration: International Standard Randomized Controlled Trial Number (ISRCTN) 15394285; http://www.isrctn.com/ISRCTN15394285

International Registered Report Identifier (IRRID)： DERR1-10.2196/21116

(JMIR Res Protoc 2021;10(3):e21116) doi: 10.2196/21116

\section{KEYWORDS}

type 2 diabetes; diabetes; diabetic diet; low-energy diet; low calorie diet; intermittent energy restriction; intermittent fasting; diabetes remission; smartphone; mobile phone; mHealth; mobile health

\section{Introduction}

\section{Background}

An estimated 4.7 million people have type 2 diabetes (T2D) in the United Kingdom, with the number expected to rise to over 5.5 million by 2030 [1]. Diabetes related complications are common, and people with T2D die up to 10 years earlier than those without the disease [2]. Currently, $10 \%$ of the National Health Service (NHS) budget in the United Kingdom is spent on diabetes (approximately $£ 10$ billion (US $\$ 13.8$ billion) per year) [1].

Approximately $80 \%$ to $90 \%$ of people with T2D have overweight or obesity [3-6]. Clinical guidelines for the management of T2D focus largely on multiple drug treatments to reduce blood glucose. They also recommend at least $5 \%$ to $10 \%$ weight loss $[7,8]$ as this leads to improvements in glycemic control, insulin sensitivity, blood lipids, and blood pressure (BP) $[9,10]$.

\section{Continuous and Intermittent Low-Energy Diets}

Intensive face-to-face weight loss programs using continuous low-energy diets (CLEDs) that provide approximately $800 \mathrm{kcal}$ $(3347 \mathrm{~kJ})$ per day of formula-based total diet replacement for 8 to 20 weeks or longer are highly effective for large weight loss and remission from T2D [11-14]. The recently published Diabetes Remission Clinical Trial (DiRECT) tested an intensive CLED program in primary care and found that it was superior to standard best practice care (standard daily moderate energy restriction advice with minimal support). At 12 months, $45.6 \%$ (68/149) participants of the intervention group achieved remission with an average weight loss of $10 \mathrm{~kg}$ compared with $4.0 \%(6 / 149)$ and $1 \mathrm{~kg}$ loss in the control group $(P<.001)$. Remission was highest $(31 / 36,86 \%)$ in those who achieved $15 \%$ weight loss [13] and is more likely in those diagnosed with T2D in more recent years [15].

The CLED approach using total diet replacement is thought to be effective because the initial rapid weight loss can be highly motivating [16], and formula diets remove decision making around food choices. In addition, subjective hunger may be reduced by the associated ketosis [17]. CLEDs have been shown to reduce excess fat in the liver and pancreas in patients with $\mathrm{T} 2 \mathrm{D}$, which is part of the proposed mechanism for T2D remission [11,18].

Possible drawbacks of the CLED approach are that it is not appealing to or achievable for everyone. Participants following CLED programs report shame and awkwardness in social situations centered around food [19]. Attrition in intensive
CLED studies on people with overweight or obesity (+/-T2D) is approximately $25 \%[13,20]$, although higher rates have been reported in studies with less frequent health care professional (HCP) contact [14]. The prevention of weight gain following a CLED program remains a key challenge. In 2 years, participants in the CLED group in the DiRECT trial appeared to have regained approximately $40 \%$ of the weight they had lost after the initial total diet replacement phase despite regular face-to-face support and an intensive 2-year relapse program involving repeated spells of a CLED, partial meal replacements, and antiobesity medications [13,21].

An intermittent low-energy diet (ILED) is a potential alternative to CLED. This includes the same number of low-energy formula diet days as CLED, but these days are undertaken for 2 days per week over a longer period. ILED may provide an alternative approach for people who find CLEDs unappealing or difficult to maintain. Qualitative reports on people following CLEDs suggest that they would prefer an intermittent approach that may be easier to fit into life without the need for weeks away from normal food [22].

A recent randomized controlled trial (RCT) in people with T2D compared an ILED with regular, daily, modest energy restriction (1200-1500 kcal/5020-6276 kJ per day) for 12 months and showed similar improvements to glycemic control in both groups [23]. Early studies of CLEDs show that some of the improvements in insulin sensitivity and beta cell function are associated with acute energy restriction rather than weight loss [24]. As these benefits subsequently attenuate when subjects enter weight maintenance with euenergetic feeding [25], ongoing spells of intermittent energy restriction each week may be a way to maintain beneficial glycemic control. A recent RCT $(n=46)$ of participants with prediabetes and overweight or obesity showed that an ILED every other day for 12 months produced greater reductions $(P<.05)$ in fasting insulin $-52 \%$ (SE 9\%) and insulin resistance -53\% (SE 9\%) compared with isocaloric daily moderate calorie restriction $(-14 \%$, SE 9\%; $-17 \%$, SE $11 \%$ ) despite similar decreases in body weight [26].

An ILED with 2 low-energy days per week will lead to a slower initial weight loss compared with a CLED. An unanswered research question is whether an ILED may lead to improved weight loss maintenance compared with daily dietary approaches in patients with T2D.

The relative benefits of ILED versus isocaloric CLED regarding glycemic control, diabetes remission, and weight loss maintenance in people with T2D are unknown. 


\section{Remote Follow-up}

A potential strategy for increasing adherence efficacy and reach of low-energy diet (LED) programs may be to include high-frequency remote follow-up, which has been shown to be superior to low frequency face-to-face care in weight management interventions [27]. Remote care reduces participants' burden of attending face-to-face appointments and may be cost-effective compared with face-to-face care [28] while improving access to care. There is growing evidence to support the use of telehealth (including telephone and mobile phone-based apps) to monitor and provide feedback to patients with T2D and promote self-management of their condition [29-31].

The Manchester Intermittent versus Daily Diet Diabetes App Study (MIDDAS) incorporated high-frequency remote follow-up via the Oviva smartphone app and by telephone [32]. The app was used to facilitate self-monitoring of diet, weight, and blood glucose, and communication with HCPs, with the option of remote peer group support. Group participation in mobile apps has been shown to predict weight loss success [33]. Remote communities can be encouraging, motivating, and informative while remaining convenient and anonymous [34].

\section{Goals of This Study}

The primary aim of MIDDAS is to assess the feasibility and potential efficacy of remotely supported ILED and isocaloric CLED programs in patients with overweight or obesity and T2D. The feasibility of an RCT comparing the 2 approaches was also assessed. MIDDAS did not have a control group for comparison because CLED programs have already been shown to be superior to standard or best practice.[13] The initial estimates of acceptability (uptake and retention) and potential efficacy (change in weight and glycated hemoglobin $\left[\mathrm{HbA}_{1 \mathrm{c}}\right]$ ) of the programs will determine whether progression to a full RCT is indicated and will inform the feasibility of delivering ILED and CLED programs that incorporate remote follow-up.

\section{Methods}

The trial protocol (V5.0/08.04.19) was granted ethical approval by the North West Greater Manchester South Research Ethics
Committee (ref:17/NW/0389). SPIRIT reporting guidelines were used [35].

\section{Design and Setting}

The study was a 12-month pilot 2-arm RCT, performed in patients with T2D and overweight or obesity, recruited from general practices, NHS hospital trusts and an NHS-supported voluntary research register in England. Participants attended trial assessments at Manchester University NHS Foundation Trust (MFT), Manchester, United Kingdom. Participants received dietary support remotely via the Oviva app and/or by telephone.

\section{Recruitment}

Potential eligible participants were recruited from 3 settings using the following methods:

1. Patients in three general practices in Manchester were sent targeted invite letters and a text message reminder 8 weeks later if there was no response. These practices included a population of patients with T2D ranging from 400 to 700 and reflected different levels of deprivation in England. The England Index of Multiple Deprivation (IMD) score for each practice was 10.9 (least deprived 25\% in England) and 29.8 and 44.1 (top 25\% deprived in England) [36].

2. Patients at MFT and Stockport NHS Foundation Trust were invited to the study via patient record search and invitation letter, using poster displays, or during face-to-face routine clinical contacts.

3. Patients with T2D $(n=2500)$ on the Help BEAT Diabetes volunteer database (hosted by the National Institute of Health Research Clinical Research Network for Greater Manchester) were contacted via mail or email and asked to check if they met the eligibility criteria for the trial and to contact the MIDDAS trial team if they were interested in taking part.

Those invited by letter had the opportunity to tell the trial team why they did not wish to take part via an anonymous reply slip. Interested patients were invited to an optional group session at MFT to receive more information on the diets and try the Optifast meal replacements before deciding to participate.

The inclusion and exclusion criteria for the trial is detailed in Textbox 1. 
Textbox 1. Inclusion and exclusion criteria.

Inclusion criteria

- Willing and able to provide written informed consent

- Male or female aged between 18 and 75 years

- $\quad$ Diagnosed with type 2 diabetes $<8$ years

- Diet controlled only or receiving any type of diabetes medications including insulin

- Glycated hemoglobin $\left(\mathrm{HbA}_{1 \mathrm{c}}\right) \geq 48 \mathrm{mmol}$ per mol (6.5\%) at baseline (venous blood sample)

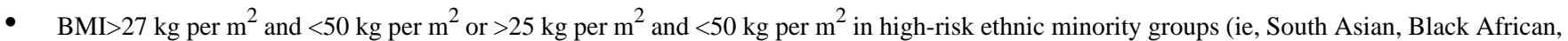
and African Caribbean)

- Access to and ability to use the telephone

- Willing to be randomized to an intermittent low-energy diet or continuous low-energy diet using total diet replacement drinks

Exclusion criteria

- $\quad$ Routine $\mathrm{HbA}_{1 \mathrm{c}} \geq 108 \mathrm{mmol}$ per mol (12.0\%) during the last 3 months

- Unstable retinopathy, grade R2 or higher, or no retinopathy screen within the last 12 months

- Pregnant or considering pregnancy

- $\quad$ Previous bariatric surgery

- $\quad$ Current treatment with Orlistat

- Unintentional weight loss $\geq 5 \mathrm{~kg}$ within last 6 months

- $\quad$ Learning difficulties, lacking capacity or unable to understand English

- Known sensitivity to ingredients in the total diet replacement

- Diagnosed eating disorder. Severe binge eating or very low eating self-efficacy were assessed using the following questionnaires: Binge Eating Scale (BES [37], score $\geq 27$ ) and Weight Efficacy Lifestyle Questionnaire Short Form (WEL-SF [38], score $\leq 35$ )

- Severe anxiety or depression was assessed using the Generalized Anxiety Disorder Scale (GAD-7 [39], score $\geq 15$ ) and Patient Health Questionnaire-9 (PHQ-9[40], score $\geq 15$ ). Hazardous or harmful drinking was indicated by the Alcohol Use Disorders Identification Test (AUDIT [41], score $\geq 16)$

- Active symptoms associated with emotionally unstable personality disorder, bipolar disorders, psychotic disorders, post-traumatic stress disorder, and current self-harm or suicidal behavior. Participants with these issues were potentially eligible, dependent on further information from their general practitioners and responses to the baseline study questionnaires

- Current treatment with lithium, antipsychotics, or other psychotropic medications that may cause excessive weight gain

- Chronic use of steroids

- Medical conditions that in the opinion of the treating physician were at risk of deterioration (eg, severe systemic or organ disease, active cancer, liver, gall bladder disease, and pancreatitis)

- Current participation in a diabetes drug trial

\section{Participant Flow and Medication Management}

The participant flow through the study is outlined in Figure 1. Informed consent was obtained by the trial research nurse at the baseline appointment. Eligible participants were randomized to ILED or CLED. All participants were invited to attend follow-up appointments at MFT for a repeat of clinical measurements at weeks $8,12,28$, and 52 . 
Figure 1. Participant flow through study.

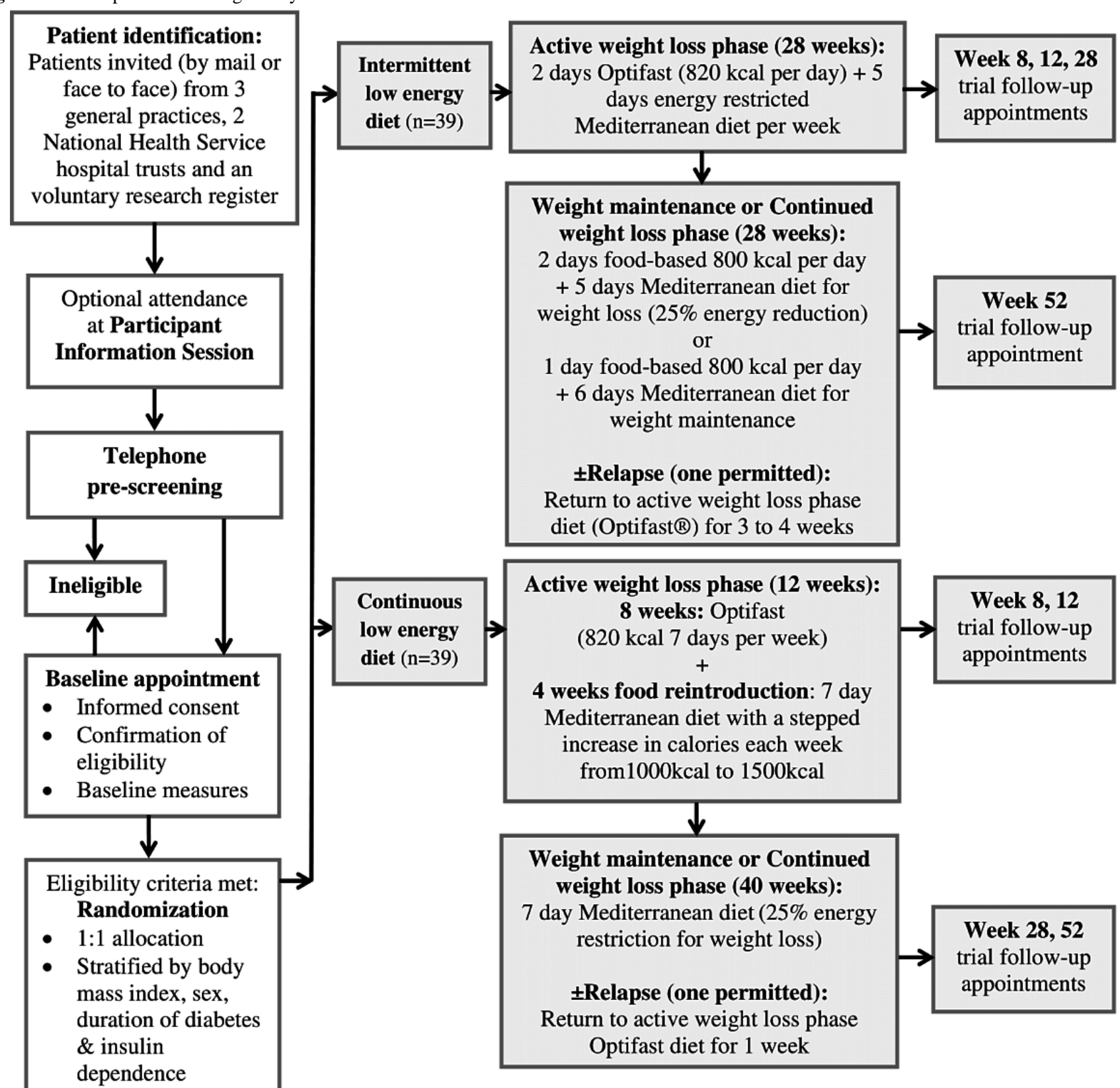

\section{Recruitment}

Changes to diabetes and antihypertensive medications specific to each treatment arm are detailed in the trial medication management plan in Table S2 of Multimedia Appendix 1. The CLED medication management protocol was devised by the research team and instructed participants to stop all diabetes medications, with the exception of metformin. Insulin was stopped or reduced, depending on the baseline $\mathrm{HbA}_{1 \mathrm{c}}$ level. Medication management of the ILED arm was adapted from a protocol tested in a recent ILED trial [42]. Medications in both arms were reintroduced or titrated during the trial according to clinical needs. General practitioners (GPs) were notified of the enrollment of patients and changes to their medications by letter.

\section{Intervention (52 weeks)}

\section{Randomization and Blinding}

Eligible participants were randomized 1:1 to ILED or CLED by a researcher independent of the intervention using a minimization program stratified by BMI $\geq 34$ or $<34 \mathrm{~kg}$ per $\mathrm{m}^{2}$ (projected mean value from the counterpoint and counterbalance studies $[11,12,15])$, sex, duration of diabetes $<4$ years or $\geq 4$ years, and whether participants were prescribed insulin. Due to the nature of the intervention, it was not possible to blind participants and clinicians to the treatment allocation. Clinical assessments were performed by an independent research assistant. Laboratory results were assessed by independent laboratory staff. 


\section{Interventions}

Figure 1 shows a summary of the 2 dietary programs. Both included a combination of the Optifast LED and the Mediterranean diet over a period of 12 months. Both programs were designed to have exactly 56 Optifast LED days and an equivalent level of energy restriction during their active weight loss phase and weight maintenance or continued weight loss phase.

\section{CLED}

\section{Active Weight Loss Phase (12 weeks)}

Weeks 1 to 8 involved the Optifast LED (Néstle Health Science, United Kingdom). This provided approximately $820 \mathrm{kcal}$ (3430 $\mathrm{kJ})$ per day and consisted of 3 sachets per day of Optifast (200 $\mathrm{kcal} / 837 \mathrm{~kJ}$ per sachet of shake or soup made with water) in addition to $8 \times 80 \mathrm{~g}$ portions of nonstarchy vegetables (approximately $140 \mathrm{kcal} / 586 \mathrm{~kJ}$ ), one dessertspoon of oil per day (80 kcal per $335 \mathrm{~kJ})$, and 2-2.5 liters of calorie-free fluids. The participants were asked to avoid alcohol and excessive caffeine to minimize the risk of dehydration. Participants who were unable to tolerate Optifast were offered a food-based LED with a similar macronutrient profile. This comprised $250 \mathrm{~g}$ of lean protein foods (eg, lean meat, fish, eggs, and vegetarian proteins), 5 portions of nonstarchy vegetables, 3 portions of low-fat dairy (eg, $200 \mathrm{ml}$ milk), one portion of unsaturated fat (eg, small handful nuts), one portion of fruit, and one carbohydrate portion (eg, slice of bread). Participants reported their adherence to the LED days throughout the trial during their regular contact with the dietitian.

Diet reintroduction in weeks 9-12 allowed a food-based, energy-restricted Mediterranean diet providing $1000 \mathrm{kcal}$ (4184 $\mathrm{kJ})$ daily in week 1, $1200 \mathrm{kcal}(5021 \mathrm{~kJ})$ daily in week 2, 1400 $\mathrm{kcal}(5858 \mathrm{~kJ})$ daily in week 3 , and $1500 \mathrm{kcal}(6276 \mathrm{~kJ})$ daily in week 4.

The Mediterranean diet, as described previously by Harvie et al [43], was relatively high in protein (25\% energy) with moderate carbohydrate ( $45 \%$ energy from low glycemic load carbohydrates), moderate fat $(30 \%$ energy from fat: $15 \%$ monounsaturated, $8 \%$ polyunsaturated, and $7 \%$ saturated fatty acids), and limited alcohol to $<10$ units per week.

\section{Weight Maintenance or Continued Weight Loss Phase (40 Weeks)}

Participants who achieved a trial weight loss goal of $15 \%$, and/or their target weight if greater, were advised to follow a euenergetic Mediterranean diet for weight maintenance. Those who had not achieved $15 \%$ weight loss or wished to lose more weight were asked to follow a $25 \%$ energy-restricted Mediterranean diet. Individuals' estimated energy requirements were calculated using the Mifflin equations [44] to estimate the basal metabolic ratexreported metabolic equivalent of the task. These calculations were based on the revised weight and activity levels after the completion of the active weight loss phase.

\section{ILED}

\section{Active Weight Loss Phase (28 weeks)}

The ILED group was asked to include 2 consecutive days per week of the Optifast LED plus 5 days of a Mediterranean diet for 28 weeks. An energy deficit was applied to the Mediterranean diet (up to a value of $265 \mathrm{kcal}$ [1109 kJ] per day) to ensure that the ILED and CLED diets were isocaloric during the active weight loss phase.

\section{Weight Maintenance or Continued Weight Loss Phase (24 weeks)}

Participants were asked to follow a food-based ILED. Participants who had achieved $15 \%$ weight loss, and/or their target weight if greater, were asked to follow the food-based LED described above for one day per week and a euenergetic Mediterranean diet aimed at weight maintenance (eg, $2550 \mathrm{kcal}$ [10,669 kJ] per day for a 50-year-old man, BMI 34) for 6 days. Participants who had not achieved $15 \%$ weight loss or wished to lose more weight were asked to follow the food-based LED for 2 consecutive days per week and an energy-restricted Mediterranean diet (eg, $2100 \mathrm{kcal}$ [8786 Kj] per day for a 50 -year-old man, BMI 34) for 5 days. This provided an overall daily $25 \%$ energy restriction through the week.

\section{Relapse}

Trial participants who regained $2 \mathrm{~kg}$ or more during their weight maintenance or continued weight loss phase were offered one opportunity to resume their initial active weight loss diet to help reverse the weight gain. The CLED group was offered the 820 kcal $(3430 \mathrm{~kJ})$ formula Optifast LED for one week, and the ILED group was offered the Optifast LED 2 days per week for 3 to 4 weeks. The relapse program included increased support and monitoring by the trial dietitian and support from the trial psychologist to explore reasons for weight regain and to prevent further relapse.

\section{Dietitian and Nurse}

The diabetes specialist dietitian provided education on the diets and how to use the Oviva app, either by telephone or face-to-face. All participants were provided with written support materials, including recipes and meal plans. The diabetes specialist nurse provided support for patients who were on diabetes medications other than metformin, on antihypertensives, or who were hypertensive at baseline. All changes to diabetes and antihypertensive medications were agreed upon by the trial doctor and communicated to participants and their GP by a diabetes specialist nurse.

\section{Physical Activity}

Participants were encouraged to undertake physical activity (PA) throughout the trial to limit the loss of fat-free mass and promote weight loss maintenance. PA was supported by an exercise specialist, and suitability for PA was assessed at baseline using the Physical Activity Readiness Questionnaire (PAR-Q) [45] and signed off by the trial consultant endocrinologist. Participants were encouraged to aim for $5 \times 30$ minutes of moderate-intensity cardiovascular PA per week and resistance exercises for the legs, arms, and trunk 3 times per week. They were signposted to local PA services as appropriate 
and were educated on minimizing the risk of hypoglycemia. Diabetes medications were managed alongside exercise in response to the reported blood glucose readings.

\section{Psychological Support}

Enhanced psychological support from the trial psychologist was available for participants whose baseline scores indicated moderate scores for binge eating (score 18-26 on BES [37]), self-efficacy (score 36-45 on the Weight Efficacy Lifestyle Questionnaire Short Form (WEL-SF) [38]), anxiety (score 10-14 on the generalized anxiety disorder scale (GAD-7, [39]), depression (score 10-14 on the Patient Health Questionnaire-9 [PHQ-9]; [40]), or risk of alcohol-related problems (score 8-15 on the Alcohol Use Disorders Identification Test [AUDIT]; [41]). Psychological support was also available to participants who relapsed or had been identified by the team as experiencing difficulties impairing their ability to adhere to the programs. Psychological intervention was informed by motivational interviews, cognitive behavioral therapy, behavioral activation, mindfulness, emotional regulation, and distress tolerance skills.

\section{Remote Behavioral Support}

All participants received regular remote support from a multidisciplinary team including a diabetes specialist dietitian, nurse, and exercise specialist. The frequency and mode of behavioral support are detailed in Tables S1 and S2 in Multimedia Appendix 2. For example, the dietitian contacted the participants weekly via Oviva app messaging in weeks 1 to 12 , fortnightly in weeks 13 to 28, and monthly in weeks 29 to 52 . Follow-up telephone calls with the dietitian were performed at weeks $8,12,28$, and 52 .

Participants were invited to communicate with the multidisciplinary team via the Oviva app functional on iOS and Android smartphones and tablets. The app facilitates written messages and self-monitoring of diet, weight, blood glucose, weight, and activity levels. Participants were also invited to take part in group messaging on the app with other participants from their allocated diet group. Use of the app was optional, so if participants chose not to use it, then their scheduled contacts were done by telephone.

Participants signed a treatment contract to monitor their blood glucose and BP according to the protocol (Table S1 in Multimedia Appendix 1) and reported these values by telephone or via the Oviva app to the multidisciplinary team. The number of participants in both arms who requested face-to-face dietary consultations during the trial was recorded.

The multidisciplinary team was trained in motivational interview techniques (a well-established model of supporting behavioral change with proven efficacy in facilitating weight loss) to support dietary behavioral changes during both LED programs and in the longer term. Both programs used behavior change techniques such as problem solving and feedback on behavior, identified in a recent systematic review as being effective in reducing $\mathrm{HbA}_{1 \mathrm{c}}$ [46]. The programs also used established behavior change techniques, such as goal setting and self-monitoring [47].

\section{Outcomes}

\section{Primary}

1. Uptake: To achieve an uptake of at least $10 \%$ from a primary care mail out.

2. The proportion of subjects in the ILED and CLED groups who successfully lost and maintained $>15 \%$ weight loss at 12 months, as determined by intention-to-treat (ITT) analysis.

3. The proportion of subjects in both groups who achieved $\mathrm{HbA}_{1 \mathrm{c}}<48 \mathrm{mmol}$ per mol $(6.5 \%)$ at 12 months using ITT analysis.

4. Retention: Aiming for a retention of $60 \%$ (48/79) completion as measured by attendance at the 12-month appointment. This is the acceptable completion rate in NICE guidance for commissioning weight management services in England [48].

\section{Secondary}

\section{Process Measures}

1. Participant adherence to the protocol including self-reported adherence to LED days, preference for face-to-face contact with the dietitian, preference for food-based LED days over Optifast, and attendance at follow-up appointments.

2. Download and usage of the Oviva app for self-monitoring.

\section{Exploratory Measures}

Change in the following measures across the 12-month study period:

1. Body fat and fat-free mass (bioelectrical impedance)

2. Waist and hip circumference

3. BP, lipid profile, and fasting blood glucose levels

4. Number and dosage of diabetes and BP medications

5. Self-efficacy for eating, anxiety, depression, and quality of life (as measured by WEL-SF [38], GAD-7 [39], PHQ-9 [40] and Audit of Diabetes-Dependent Quality of Life (ADDQoL) [49] questionnaires, respectively)

6. Quality of diet on non-LED days (as measured by the Mediterranean diet score questionnaire [50])

7. PA (as measured by the Scottish Physical Activity Questionnaire [51])

8. Self-reported satisfaction with weight loss was measured using a 7-point Likert scale. This is highly relevant for the comparison of ILED and CLED, where the CLED will experience faster initial weight loss and achievement of their weight loss goal than the ILED arm [52]

9. Serious adverse events reported up to the end of the 12-month trial

\section{Measurements}

Table 1 provides a summary of the measurements collected at baseline and weeks $8,12,28$, and 52 by a research nurse. 
Table 1. Schedule of enrollment and assessments.

\begin{tabular}{|c|c|c|c|c|c|}
\hline \multirow[t]{2}{*}{ Schedule } & \multirow{2}{*}{$\begin{array}{l}\text { Enrollment } \\
\text { Baseline }\end{array}$} & \multicolumn{4}{|c|}{ Follow-up visits } \\
\hline & & 8 weeks & 12 weeks & 28 weeks & 52 weeks \\
\hline \multicolumn{6}{|l|}{ Enrollment } \\
\hline Informed consent & $\mathfrak{S}^{\mathrm{a}}$ & $-\mathrm{b}$ & - & - & - \\
\hline Eligibility screen & $\checkmark$ & - & - & - & - \\
\hline Randomization & $\checkmark$ & - & - & - & - \\
\hline \multicolumn{6}{|l|}{ Assessments } \\
\hline Height & $\checkmark$ & - & - & - & - \\
\hline Weight & $\checkmark$ & $\checkmark$ & $\checkmark$ & $\checkmark$ & $\checkmark$ \\
\hline Waist circumference & $\checkmark$ & $\checkmark$ & $\checkmark$ & $\checkmark$ & $\checkmark$ \\
\hline Hip circumference & $\checkmark$ & $\checkmark$ & $\checkmark$ & $\checkmark$ & $\checkmark$ \\
\hline Body fat or fat-free mass (impedance) & $\checkmark$ & $\checkmark$ & $\checkmark$ & $\checkmark$ & $\checkmark$ \\
\hline Blood Pressure, heart rate ${ }^{c}$ & $\checkmark$ & $\checkmark$ & $\checkmark$ & $\checkmark$ & $\checkmark$ \\
\hline Blood lipids, liver function, renal profile & $\checkmark$ & - & - & $\checkmark$ & $\checkmark$ \\
\hline Fasting plasma glucose & $\checkmark$ & $\checkmark$ & $\checkmark$ & $\checkmark$ & $\checkmark$ \\
\hline Laboratory $\mathrm{HbA}_{1 \mathrm{c}}{ }^{\mathrm{d}}$ & $\checkmark$ & - & $\checkmark$ & $\checkmark$ & $\checkmark$ \\
\hline Pregnancy urine test & $\checkmark$ & $\checkmark$ & $\checkmark$ & $\checkmark$ & $\checkmark$ \\
\hline $\mathrm{BES}^{\mathrm{e}}$ questionnaire & $\checkmark$ & - & - & $\checkmark$ & $\checkmark$ \\
\hline AUDIT $^{\mathrm{f}}$ questionnaire & $\checkmark$ & - & - & $\checkmark$ & $\checkmark$ \\
\hline WEL-SF ${ }^{\mathrm{g}}$ questionnaire & $\checkmark$ & - & - & $\checkmark$ & $\checkmark$ \\
\hline PHQ-9 ${ }^{\mathrm{h}}$ questionnaire & $\checkmark$ & $\checkmark$ & $\checkmark$ & $\checkmark$ & $\checkmark$ \\
\hline GAD- $7^{\mathrm{i}}$ questionnaire & $\checkmark$ & $\checkmark$ & $\checkmark$ & $\checkmark$ & $\checkmark$ \\
\hline EQ-5D-3L $\mathrm{L}^{\mathrm{j}}$ questionnaire & $\checkmark$ & - & - & $\checkmark$ & $\checkmark$ \\
\hline ADDQoL $^{\mathrm{k}}$ questionnaire & $\checkmark$ & - & - & $\checkmark$ & $\checkmark$ \\
\hline Mediterranean diet score & $\checkmark$ & - & $\checkmark$ & $\checkmark$ & $\checkmark$ \\
\hline S-PAQ ${ }^{1}$, PAR-Q $^{\mathrm{m}}$ & $\checkmark$ & - & $\checkmark$ & $\checkmark$ & $\checkmark$ \\
\hline Self-satisfaction with the weight loss question ${ }^{\mathrm{n}}$ & - & $\checkmark$ & $\checkmark$ & $\checkmark$ & $\checkmark$ \\
\hline Participant qualitative interviews & - & - & $\checkmark\left(\mathrm{CLED}^{\mathrm{o}}\right)$ & $\checkmark\left(\operatorname{ILED}^{\mathrm{p}}\right)$ & - \\
\hline Health care professional qualitative interviews & - & - & - & - & $\checkmark$ \\
\hline
\end{tabular}

${ }^{a}$ Event or assessment occurred at this time point.

${ }^{b}$ Event or assessment did not occur at this time point.

${ }^{\mathrm{c}}$ Further investigation with an ECG if heart rate $<50$ beats per minute and not on beta-blockers.

${ }^{\mathrm{d}} \mathrm{HbA}_{1 \mathrm{c}}$ : glycated hemoglobin.

${ }^{\mathrm{e}}$ BES: Binge Eating Scale.

${ }^{f}$ AUDIT: Alcohol Use Disorders Identification Test.

${ }^{\mathrm{g}}$ WEL-SF: Weight Efficacy Lifestyle Questionnaire Short Form.

${ }^{\mathrm{P}} \mathrm{PHQ}-9$ : Patient Health Questionnaire scale-9.

${ }^{\mathrm{i}}$ GAD-7: Generalized Anxiety Disorder scale-7.

${ }^{j}$ EQ-5D-3L: Measure of health-related quality of life.

${ }^{k}$ ADDQoL: Audit of Diabetes-Dependent Quality of Life.

${ }^{1}$ S-PAQ: Scottish Physical Activity Questionnaire.

mPAR-Q: Physical Activity Readiness Questionnaire.

${ }^{\mathrm{n}}$ Given the effort you put into following the diet and exercise plan, how satisfied are you with the amount of weight you have lost or gained during the 
past month? 1=very dissatisfied to $7=$ very satisfied.

${ }^{\circ}$ CLED: continuous low-energy diet.

PILED: intermittent low-energy diet.

\section{Participant Characteristics}

At baseline, we collected information on participants' age, sex, marital status, number of dependents living at home, ethnicity, education history and employment status, IMD score based on their postal code, relevant medical history, and current medications.

\section{Physical Measurements}

Weight and body composition were measured using Tanita BC-300MA calibrated scales to the nearest $0.1 \mathrm{~kg}$. Height was measured using a portable stadiometer. Waist circumference was measured halfway between the point of the lowest rib and the iliac crest, and hip circumference was measured at the maximum circumference of the buttocks [53]. All measurements were taken to the nearest $1 \mathrm{~mm}$. BP was measured with patients seated at rest for at least 10 minutes. All assessors were trained in accordance with the departmental protocols.

\section{Fasting Blood Sample}

A fasting venous blood sample was collected for $\mathrm{HbA}_{1 \mathrm{c}}$, plasma glucose, lipid profile, serum urea and electrolytes, eGFR, creatinine, and liver function tests. A urine pregnancy test was performed in women of childbearing age to exclude pregnancy. Pregnant women were excluded from the trial. Laboratory results were assessed by independent laboratory staff.

\section{Questionnaires}

The full list of questionnaires referenced in Table 1 is BES [37], WEL-SF [38], GAD-7 [39], PHQ-9 [40], AUDIT [41], PAR-Q [45], ADDQoL [49], Mediterranean diet score [50], Scottish Physical Activity Questionnaire [51], and EQ-5D-3L [54] (standardized validated instrument used to measure general health status). All questionnaires for which a third party does not own the copyright can be found in Multimedia Appendices 3 [37], 4 [38], 5 [39], 6 [40], 7 [41], 8 [45], 9 [50], 10 [51], and 11 [54].

\section{Retention and Withdrawal}

Participants had the right to withdraw from the trial at any time. Participants were considered as withdrawn from the trial if they withdrew from the study intervention voluntarily or if they failed to return for follow-up assessments. Participants could also be removed by the principal investigator if this was considered necessary for medical reasons or due to ineligibility arising during the study (eg, pregnancy). Reasons for withdrawal were recorded, and their GP was notified with recommendations for follow-up care where appropriate. Participants who withdrew continued to have weight and $\mathrm{HbA}_{1 \mathrm{c}}$ collected from their routine diabetes clinic or GP visits for the duration of the 12-month trial unless they did not consent to this at baseline. No incentives were provided to the participants to promote retention and follow-up.

\section{Adverse Events}

All adverse events in the 12-month study period were recorded by following the Good Clinical Practice and Health Research Authority processes. Nonserious adverse events such as constipation, fatigue, or hair loss were recorded when participants informed the trial team.

\section{Qualitative Evaluation}

In-depth semistructured interviews were conducted with a subset of 10 ILED and 10 CLED participants at the end of the active weight loss phase (ILED week 28, CLED week 12). HCPs ( $n=6)$ delivering the programs were also interviewed near study completion. All interviews were conducted by an independent research assistant trained in qualitative interview techniques. Interviews explored the participant and HCP experiences of the ILED and CLED programs and the use of the Oviva app. Purposive sampling was used to select trial participants with a range of success in terms of actual weight loss and $\mathrm{HbA}_{1 \mathrm{c}}$ reduction, as well as participants' perceived success. Participants with ethnicities other than White-British were included where possible, with a fairly even split between men and women. All participants gave written informed consent to be interviewed and were assured that their data would be anonymized. The interviews were audiorecorded and transcribed verbatim for thematic analysis.

\section{Sample Size and Statistical Analysis}

The total number of participants recruited for the study was 79 . The sample size was selected to allow an estimate of uptake within $\pm 6.6 \%$ of the target uptake of $10 \%$ [55] with $95 \%$ confidence while allowing the research team to obtain sufficient data on the feasibility and potential efficacy of the ILED and CLED.

This study will not undertake significance tests of changes to the primary outcome measures. Descriptive, graphical (summary), and basic inferential statistics of outcomes will be presented as appropriate, for example, frequencies and percentages, mean and SD, or median and quartiles. Confidence intervals $(95 \%)$ will be calculated to show the change from baseline in the outcomes for each group.

Questionnaires used as outcome measures are quantitative and will be analyzed using appropriate descriptive statistics as per standard.

Changes to diabetes medication will be presented using the medication effect score (MES). The MES for a participant is the sum of the MES for each of their individual medications, where $\mathrm{MES}=$ actual drug dose/maximum drug dosexdrug mean adjustment factor. A decrease in MES corresponds to a decrease in the use of diabetes medications [56]. Changes to BP medications will be presented using a Treatment Intensity Score (TIS) defined as the actual drug dose/maximum drug dose [57]. The TIS for a participant is the sum of the TIS for each of their medications, and a decrease in TIS indicates a decrease in BP medications. 
An ITT analysis using multiple imputations will conducted for percentage weight loss, $\mathrm{HbA}_{1 \mathrm{c}}$, and MES. All other outcomes will be presented only for those who completed the trial.

\section{Data Management}

Data were recorded on hard copy case report forms and subsequently transferred to a database with ranges and programmed validation checks to aid reliable data entry. Data are held on secure servers at MFT.

\section{Trial Steering Committee and Trial Management Group}

The trial steering committee provided oversight for participant safety and included 2 co-principal investigators (BI and $\mathrm{MH}$ ), an external endocrinologist and independent external advisor with experience of LEDs in the management of T2D. The committee met every 3 months to review and ensure the safety aspects of the trial. The trial management group, which comprised the chief investigators, diabetes specialist dietitians, a diabetes specialist nurse, and research nurses, evaluated all adverse events. The trial could have been stopped by the sponsor, chief investigators, or the trial management group or trial steering committee on the basis of new safety information or for other reasons given by the research ethics committee, but this was not required. The trial was subject to inspection and audit by MFT as the trial sponsor.

\section{Results}

The project was funded in May 2017, ethical approval was obtained in August 2017, and enrollment began in February 2018. In total, 79 participants were recruited and randomized to the ILED $(n=39)$ and CLED $(n=40)$ arms of the trial. Data collection was completed in February 2020. Data analysis began in June 2020, and the first results are expected to be submitted for publication in 2021 .

\section{Discussion}

This is the first study to compare an ILED with an isocaloric CLED to achieve and maintain weight loss and normoglycemia among patients with T2D and overweight and obesity. The study will inform the acceptability and potential efficacy of high-frequency remote follow-up in patients with T2D and overweight and obesity undertaking low energy diets. It will also contribute to the limited data on the safety and efficacy of patients with T2D on insulin undertaking a ILED or CLED. The study did not have a standard or best practice control group for comparison and was not powered to show statistical differences between the groups. However, the planned quantitative and qualitative analyses will assess the feasibility of the programs and inform the case for a future definitive trial.

\section{Acknowledgments}

This work was supported by Néstle Health Science and Oviva UK Limited. Oviva also contributed to the study design. There are no restrictions on the publication of the results of this study, and the contract between the funder and the sponsor (MFT, Research and Innovation, Wythenshawe Hospital, M23 9LT) ensures that the funding body will have no say in the decisions regarding publication. The authors would like to thank Bowland Medical Practice, Northenden Group Practice, and Washway Road Medical Centre for their role in recruitment; John Belcher for his invaluable advice on the statistics; Lucinda Summers for her role as an external endocrinologist overseeing the safety of the study; and Cathy Spence, Schvearn Allen, and the wider MFT Research and Innovation team for their contributions to the set-up and conduct of the trial. The MIDDAS team also thank the participants for their valuable contribution to the study and without whom research of this kind would not be possible.

\section{Authors' Contributions}

MH, BI, and SM designed the study and secured the funding. SM drafted the manuscript for publication with input from MH and BI. All other authors have proofread and checked the manuscript.

\section{Conflicts of Interest}

The authors declare no conflicts of interest. Néstle Health Science, as the funder of the trial, is also the manufacturer of the nutritional products used in the trial. Oviva provided a smartphone app used in the trial.

\section{Multimedia Appendix 1}

Monitoring and medication management plan.

[DOCX File, 28 KB-Multimedia Appendix 1]

\section{Multimedia Appendix 2}

Support provided by the multidisciplinary team. [DOCX File , $21 \mathrm{~KB}-$ Multimedia Appendix 2]

\section{Multimedia Appendix 3}

Binge Eating Scale. 


\section{Multimedia Appendix 4}

Weight efficacy lifestyle questionnaire short-form.

[DOC File, 49 KB-Multimedia Appendix 4]

\section{Multimedia Appendix 5}

Generalized anxiety disorder scale.

[DOCX File, 119 KB-Multimedia Appendix 5]

\section{Multimedia Appendix 6}

Patient health questionnaire.

[DOCX File, 124 KB-Multimedia Appendix 6]

\section{Multimedia Appendix 7}

Alcohol use disorders identification test.

[DOC File, 128 KB-Multimedia Appendix 7]

\section{Multimedia Appendix 8}

Physical activity readiness questionnaire.

[DOCX File , 49 KB-Multimedia Appendix 8]

\section{Multimedia Appendix 9}

Mediterranean diet score.

[DOCX File , $31 \mathrm{~KB}-$ Multimedia Appendix 9]

\section{Multimedia Appendix 10}

Scottish physical activity questionnaire.

[DOC File, 97 KB-Multimedia Appendix 10]

\section{Multimedia Appendix 11}

Eq-5d-31.

[DOCX File, 63 KB-Multimedia Appendix 11]

\section{References}

1. Us, diabetes and lots of facts and stats 2019. Diabetes UK. URL: https://www.diabetes.org.uk/resources-s3/2019-02/ 1362B Facts\%20and\%20stats\%20Update\%20Jan\%202019 LOW\%20RES EXTERNAL.pdf [accessed 2020-05-27]

2. Nwaneri C, Bowen-Jones D, Cooper H, Chikkaveerappa K, Afolabi BA. Falling mortality rates in Type 2 diabetes mellitus in the Wirral Peninsula: a longitudinal and retrospective cohort population-based study. Postgrad Med J 2012 Dec;88(1046):679-683. [doi: 10.1136/postgradmedj-2012-130877] [Medline: 22904202]

3. Tobias DK, Pan A, Jackson CL, O'Reilly EJ, Ding EL, Willett WC, et al. Body-mass index and mortality among adults with incident type 2 diabetes. N Engl J Med 2014 Jan 16;370(3):233-244 [FREE Full text] [doi: 10.1056/NEJMoa1304501] [Medline: 24428469]

4. Adult obesity and type 2 diabetes. Public Health England. URL: https://assets.publishing.service.gov.uk/government/ uploads/system/uploads/attachment_data/file/338934/Adult_obesity_and_type_2_diabetes_pdf [accessed 2020-05-27]

5. Bray GA. Medical consequences of obesity. J Clin Endocrinol Metab 2004 Jun;89(6):2583-2589. [doi: 10.1210/jc.2004-0535] [Medline: 15181027$]$

6. Abdullah A, Peeters A, de Courten M, Stoelwinder J. The magnitude of association between overweight and obesity and the risk of diabetes: a meta-analysis of prospective cohort studies. Diabetes Res Clin Pract 2010 Sep;89(3):309-319. [doi: 10.1016/j.diabres.2010.04.012] [Medline: 20493574]

7. NG28 Type 2 diabetes in adults: management. National Institute for Health and Care Excellence. 2015. URL: https://www. nice.org.uk/guidance/ng28 [accessed 2020-05-27]

8. 115 Management of obesity :a national clinical guideline. Scottish Intercollegiate Guidelines Network. 2010. URL: https:/ /www.sign.ac.uk/assets/sign115.pdf [accessed 2020-05-27] 
9. Franz MJ, Boucher JL, Rutten-Ramos S, VanWormer JJ. Lifestyle weight-loss intervention outcomes in overweight and obese adults with type 2 diabetes: a systematic review and meta-analysis of randomized clinical trials. J Acad Nutr Diet 2015 Sep;115(9):1447-1463. [doi: 10.1016/j.jand.2015.02.031] [Medline: 25935570]

10. Albu JB, Heilbronn LK, Kelley DE, Smith SR, Azuma K, Berk ES, Look AHEAD Adipose Research Group. Metabolic changes following a 1-year diet and exercise intervention in patients with type 2 diabetes. Diabetes 2010 Mar;59(3):627-633 [FREE Full text] [doi: 10.2337/db09-1239] [Medline: 20028945]

11. Lim EL, Hollingsworth KG, Aribisala BS, Chen MJ, Mathers JC, Taylor R. Reversal of type 2 diabetes: normalisation of beta cell function in association with decreased pancreas and liver triacylglycerol. Diabetologia 2011 Oct;54(10):2506-2514 [FREE Full text] [doi: 10.1007/s00125-011-2204-7] [Medline: 21656330]

12. Steven S, Hollingsworth KG, Al-Mrabeh A, Avery L, Aribisala B, Caslake M, et al. Very low-calorie diet and 6 months of weight stability in type 2 diabetes: pathophysiological changes in responders and nonresponders. Diabetes Care 2016 May;39(5):808-815. [doi: 10.2337/dc15-1942] [Medline: 27002059]

13. Lean ME, Leslie WS, Barnes AC, Brosnahan N, Thom G, McCombie L, et al. Primary care-led weight management for remission of type 2 diabetes (DiRECT): an open-label, cluster-randomised trial. Lancet 2018 Feb 10;391(10120):541-551. [doi: 10.1016/S0140-6736(17)33102-1] [Medline: 29221645]

14. Brown A, Leeds AR. Very low - energy and low - energy formula diets: Effects on weight loss, obesity co - morbidities and type 2 diabetes remission - an update on the evidence for their use in clinical practice. Nutr Bull 2019 Feb 20;44(1):7-24. [doi: 10.1111/nbu.12372]

15. Steven S, Taylor R. Restoring normoglycaemia by use of a very low calorie diet in long-and short-duration Type 2 diabetes. Diabet Med 2015 Sep;32(9):1149-1155. [doi: 10.1111/dme.12722] [Medline: 25683066]

16. Rehackova L, Araújo-Soares V, Adamson AJ, Steven S, Taylor R, Sniehotta FF. Acceptability of a very-low-energy diet in Type 2 diabetes: patient experiences and behaviour regulation. Diabet Med 2017 Nov;34(11):1554-1567 [FREE Full text] [doi: 10.1111/dme.13426] [Medline: 28727247]

17. Sumithran P, Prendergast LA, Delbridge E, Purcell K, Shulkes A, Kriketos A, et al. Ketosis and appetite-mediating nutrients and hormones after weight loss. Eur J Clin Nutr 2013 Jul 1;67(7):759-764. [doi: 10.1038/ejcn.2013.90] [Medline: 23632752]

18. Taylor R. Pathogenesis of type 2 diabetes: tracing the reverse route from cure to cause. Diabetologia 2008 Oct;51(10):1781-1789. [doi: 10.1007/s00125-008-1116-7] [Medline: 18726585]

19. Rehackova L, Araújo-Soares V, Steven S, Adamson AJ, Taylor R, Sniehotta FF. Behaviour change during dietary Type 2 diabetes remission: a longitudinal qualitative evaluation of an intervention using a very low energy diet. Diabet Med 2020 Jun;37(6):953-962. [doi: 10.1111/dme.14066] [Medline: 31269276]

20. Astbury NM, Aveyard P, Nickless A, Hood K, Corfield K, Lowe R, et al. Doctor Referral of Overweight People to Low Energy total diet replacement Treatment (DROPLET): pragmatic randomised controlled trial. Br Med J 2018 Sep 26;362:3760 [FREE Full text] [doi: 10.1136/bmj.k3760] [Medline: $\underline{\text { 30257983] }}$

21. Lean MEJ, Leslie WS, Barnes AC, Brosnahan N, Thom G, McCombie L, et al. Durability of a primary care-led weight-management intervention for remission of type 2 diabetes: 2-year results of the DiRECT open-label, cluster-randomised trial. Lancet Diabetes Endocrinol 2019 May;7(5):344-355. [doi: 10.1016/S2213-8587(19)30068-3] [Medline: 30852132]

22. Rössner S. Intermittent vs continuous VLCD therapy in obesity treatment. Int J Obes Relat Metab Disord 1998 Feb;22(2):190-192. [doi: 10.1038/sj.ijo.0800563] [Medline: 9504328]

23. Carter S, Clifton PM, Keogh JB. Effect of intermittent compared with continuous energy restricted diet on glycemic control in patients with type 2 diabetes: a randomized noninferiority trial. JAMA Netw Open 2018 Jul 06;1(3):e180756 [FREE Full text] [doi: 10.1001/jamanetworkopen.2018.0756] [Medline: 30646030]

24. Jackness C, Karmally W, Febres G, Conwell IM, Ahmed L, Bessler M, et al. Very low-calorie diet mimics the early beneficial effect of Roux-en-Y gastric bypass on insulin sensitivity and $\beta$-cell Function in type 2 diabetic patients. Diabetes 2013 Sep;62(9):3027-3032 [FREE Full text] [doi: 10.2337/db12-1762] [Medline: 23610060]

25. Wing RR, Blair E, Marcus M, Epstein LH, Harvey J. Year-long weight loss treatment for obese patients with type II diabetes: does including an intermittent very-low-calorie diet improve outcome? Am J Med 1994 Oct;97(4):354-362. [doi: 10.1016/0002-9343(94)90302-6] [Medline: 7942937]

26. Gabel K, Kroeger CM, Trepanowski JF, Hoddy KK, Cienfuegos S, Kalam F, et al. Differential effects of alternate-day fasting versus daily calorie restriction on insulin resistance. Obesity (Silver Spring) 2019 Sep;27(9):1443-1450 [FREE Full text] [doi: 10.1002/oby.22564] [Medline: $\underline{31328895}$ ]

27. Digenio AG, Mancuso JP, Gerber RA, Dvorak RV. Comparison of methods for delivering a lifestyle modification program for obese patients: a randomized trial. Ann Intern Med 2009 Feb 17;150(4):255-262. [doi: 10.7326/0003-4819-150-4-200902170-00006] [Medline: 19221377]

28. Little P, Stuart B, Hobbs FR, Kelly J, Smith ER, Bradbury KJ, et al. Randomised controlled trial and economic analysis of an internet-based weight management programme: POWeR+ (Positive Online Weight Reduction). Health Technol Assess 2017 Jan;21(4):1-62 [FREE Full text] [doi: 10.3310/hta21040] [Medline: 28122658] 
29. Lee PA, Greenfield G, Pappas Y. The impact of telehealth remote patient monitoring on glycemic control in type 2 diabetes: a systematic review and meta-analysis of systematic reviews of randomised controlled trials. BMC Health Serv Res 2018 Jun 26;18(1):495 [FREE Full text] [doi: 10.1186/s12913-018-3274-8] [Medline: 29940936]

30. Kitsiou S, Paré G, Jaana M, Gerber B. Effectiveness of mHealth interventions for patients with diabetes: an overview of systematic reviews. PLoS One 2017;12(3):e0173160 [FREE Full text] [doi: 10.1371/journal.pone.0173160] [Medline: 28249025]

31. Bonoto BC, de Araújo VE, Godói IP, de Lemos LLP, Godman B, Bennie M, et al. Efficacy of mobile apps to support the care of patients with diabetes mellitus: a systematic review and meta-analysis of randomized controlled trials. JMIR Mhealth Uhealth 2017 Mar 01;5(3):e4 [FREE Full text] [doi: 10.2196/mhealth.6309] [Medline: 28249834]

32. Haas K, Hayoz S, Maurer-Wiesner S. Effectiveness and feasibility of a remote lifestyle intervention by dietitians for overweight and obese adults: pilot study. JMIR Mhealth Uhealth 2019 Apr 11;7(4):e12289 [FREE Full text] [doi: 10.2196/12289] [Medline: 30973338]

33. Kim H, Faw M, Michaelides A. Mobile But Connected: Harnessing the Power of Self-Efficacy and Group Support for Weight Loss Success through mHealth Intervention. J Health Commun 2017 May;22(5):395-402. [doi: 10.1080/10810730.2017.1296510] [Medline: 28339324]

34. Hwang KO, Ottenbacher AJ, Green AP, Cannon-Diehl MR, Richardson O, Bernstam EV, et al. Social support in an internet weight loss community. Int J Med Inform 2010 Jan;79(1):5-13 [FREE Full text] [doi: 10.1016/j.ijmedinf.2009.10.003] [Medline: $\underline{19945338]}$

35. Chan AW, Tetzlaff JM, Altman DG, Laupacis A, Gøtzsche PC, Krleža-Jerić K, et al. SPIRIT 2013 statement: defining standard protocol items for clinical trials. Ann Intern Med 2013 Feb 05;158(3):200-207 [FREE Full text] [doi: 10.7326/0003-4819-158-3-201302050-00583] [Medline: 23295957]

36. National General Practice Profiles 2019. Public Health England. URL: https://fingertips.phe.org.uk/profile/general-practice/ data [accessed 2020-05-27]

37. Gormally J, Black S, Daston S, Rardin D. The assessment of binge eating severity among obese persons. Addict Behav 1982;7(1):47-55. [doi: 10.1016/0306-4603(82)90024-7] [Medline: 7080884]

38. Ames GE, Heckman MG, Grothe KB, Clark MM. Eating self-efficacy: development of a short-form WEL. Eat Behav 2012 Dec;13(4):375-378. [doi: 10.1016/j.eatbeh.2012.03.013] [Medline: 23121791]

39. Spitzer RL, Kroenke K, Williams JBW, Löwe B. A brief measure for assessing generalized anxiety disorder: the GAD-7. Arch Intern Med 2006 May 22;166(10):1092-1097. [doi: 10.1001/archinte.166.10.1092] [Medline: 16717171]

40. Kroenke K, Spitzer RL, Williams JB. The PHQ-9: validity of a brief depression severity measure. J Gen Intern Med 2001 Sep;16(9):606-613 [FREE Full text] [doi: 10.1046/j.1525-1497.2001.016009606.x] [Medline: 11556941]

41. Saunders JB, Aasland OG, Babor TF, de la Fuente JR, Grant M. Development of the Alcohol Use Disorders Identification Test (AUDIT): WHO collaborative project on early detection of persons with harmful alcohol consumption-II. Addiction 1993 Jun;88(6):791-804. [doi: 10.1111/j.1360-0443.1993.tb02093.x] [Medline: $\underline{\text { 8329970] }}$

42. Carter S, Clifton PM, Keogh JB. Intermittent energy restriction in type 2 diabetes: a short discussion of medication management. World J Diabetes 2016 Dec 15;7(20):627-630 [FREE Full text] [doi: 10.4239/wjd.v7.i20.627] [Medline: 28031781]

43. Harvie M, Wright C, Pegington M, McMullan D, Mitchell E, Martin B, et al. The effect of intermittent energy and carbohydrate restriction $\mathrm{v}$. daily energy restriction on weight loss and metabolic disease risk markers in overweight women. Br J Nutr 2013 Oct;110(8):1534-1547 [FREE Full text] [doi: 10.1017/S0007114513000792] [Medline: 23591120]

44. Madden AM, Mulrooney HM, Shah S. Estimation of energy expenditure using prediction equations in overweight and obese adults: a systematic review. J Hum Nutr Diet 2016 Aug;29(4):458-476. [doi: 10.1111/jhn.12355] [Medline: 26923904]

45. Thomas S, Reading J, Shephard RJ. Revision of the Physical Activity Readiness Questionnaire (PAR-Q). Can J Sport Sci 1992 Dec;17(4):338-345. [Medline: 1330274]

46. Cradock KA, ÓLaighin G, Finucane FM, McKay R, Quinlan LR, Martin Ginis KA, et al. Diet behavior change techniques in type 2 diabetes: a systematic review and meta-analysis. Diabetes Care 2017 Dec;40(12):1800-1810. [doi: 10.2337/dc17-0462] [Medline: 29162585]

47. Michie S, Abraham C, Whittington C, McAteer J, Gupta S. Effective techniques in healthy eating and physical activity interventions: a meta-regression. Health Psychol 2009 Nov;28(6):690-701. [doi: 10.1037/a0016136] [Medline: 19916637]

48. PH53 Weight management: lifestyle services for overweight or obese adults. National Institute for Health and Care Excellence. 2014. URL: https://www.nice.org.uk/guidance/ph53/resources/ weight-management-lifestyle-services-for-overweight-or-obese-adults-pdf-1996416726469 [accessed 2020-05-27]

49. Bradley C, Todd C, Gorton T, Symonds E, Martin A, Plowright R. The development of an individualized questionnaire measure of perceived impact of diabetes on quality of life: the ADDQoL. Qual Life Res 1999;8(1-2):79-91. [doi: 10.1023/a:1026485130100] [Medline: 10457741]

50. Martínez-González MA, Fernández-Jarne E, Serrano-Martínez M, Wright M, Gomez-Gracia E. Development of a short dietary intake questionnaire for the quantitative estimation of adherence to a cardioprotective Mediterranean diet. Eur $\mathbf{J}$ Clin Nutr 2004 Nov;58(11):1550-1552. [doi: 10.1038/sj.ejcn.1602004] [Medline: 15162136] 
51. Lowther M, Mutrie N, Loughlan C, McFarlane C. Development of a Scottish physical activity questionnaire: a tool for use in physical activity interventions. Br J Sports Med 1999 Aug;33(4):244-249 [FREE Full text] [doi: 10.1136/bjsm.33.4.244] [Medline: 10450478]

52. Baldwin AS, Rothman AJ, Hertel AW, Linde JA, Jeffery RW, Finch EA, et al. Specifying the determinants of the initiation and maintenance of behavior change: an examination of self-efficacy, satisfaction, and smoking cessation. Health Psychol 2006 Sep;25(5):626-634. [doi: 10.1037/0278-6133.25.5.626] [Medline: 17014280]

53. DAPA Measurement Toolkit. Medical Research Council. URL: https://www.measurement-toolkit.org/anthropometry/ subjective-methods/introduction [accessed 2020-05-27]

54. EuroQol Group. EuroQol--a new facility for the measurement of health-related quality of life. Health Policy 1990 Dec;16(3):199-208. [doi: 10.1016/0168-8510(90)90421-9] [Medline: 10109801]

55. Justify a sample size for a feasibility study. National Institute for Health Research Research Design Service London. 2018. URL: https://www.rds-london.nihr.ac.uk/resources/justify-sample-size-for-a-feasibility-study/ [accessed 2020-05-27]

56. Mayer SB, Jeffreys AS, Olsen MK, McDuffie JR, Feinglos MN, Yancy WS. Two diets with different haemoglobin A1c and antiglycaemic medication effects despite similar weight loss in type 2 diabetes. Diabetes Obes Metab 2014 Jan;16(1):90-93 [FREE Full text] [doi: 10.1111/dom.12191] [Medline: 23911112]

57. Levy PD, Willock RJ, Burla M, Brody A, Mahn J, Marinica A, et al. Total antihypertensive therapeutic intensity score and its relationship to blood pressure reduction. J Am Soc Hypertens 2016 Dec;10(12):906-916 [FREE Full text] [doi: 10.1016/j.jash.2016.10.005] [Medline: 27856202]

\author{
Abbreviations \\ ADDQoL: Audit of Diabetes-Dependent Quality of Life \\ AUDIT: Alcohol Use Disorders Identification Test \\ BES: Binge Eating Scale \\ BP: blood pressure \\ CLED: continuous low-energy diet \\ DiRECT: Diabetes Remission Clinical Trial \\ GAD: Generalized Anxiety Disorder \\ GP: general practitioner \\ $\mathbf{H b A}_{1 \mathbf{c}}$ : glycated hemoglobin \\ HCP: health care professional \\ ILED: intermittent low-energy diet \\ IMD: Index of Multiple Deprivation \\ ITT: intention-to-treat \\ LED: low-energy diet \\ MES: medication effect score \\ MFT: Manchester University NHS Foundation Trust \\ MIDDAS: Manchester Intermittent versus Daily diet Diabetes App Study \\ NHS: National Health Service \\ PA: physical activity \\ PAR-Q: Physical Activity Readiness Questionnaire \\ PHQ-9: Patient Health Questionnaire-9 \\ RCT: randomized controlled trial \\ T2D: type 2 diabetes \\ TIS: Treatment Intensity Score \\ WEL-SF: Weight Efficacy Lifestyle Questionnaire Short Form
}

Edited by G Eysenbach; submitted 05.06.20; peer-reviewed by A Salis, A Bernier, A Coda, M Ghozali; comments to author 28.10.20;
revised version received 11.12.20; accepted 30.12.20; published 19.03.21
Please cite as:
McDiarmid S, Harvie M, Johnson R, Vyas A, Aglan A, Moran J, Ruane H, Hulme A, Sellers K, Issa B
Intermittent Versus Continuous Low-Energy Diet in Patients With Type 2 Diabetes: Protocol for a Pilot Randomized Controlled Trial
JMIR Res Protoc $2021 ; 10(3): e 21116$
URL: $\underline{\text { https://www.researchprotocols.org/2021/3/e21116 }}$
doi: $\underline{10.2196 / 21116}$
PMID: $\underline{33739297}$


(CSarah McDiarmid, Michelle Harvie, Rhona Johnson, Avni Vyas, Azza Aglan, Jacqui Moran, Helen Ruane, Amanda Hulme, Katharine Sellers, Basil Issa. Originally published in JMIR Research Protocols (http://www.researchprotocols.org), 19.03.2021. This is an open-access article distributed under the terms of the Creative Commons Attribution License (https://creativecommons.org/licenses/by/4.0/), which permits unrestricted use, distribution, and reproduction in any medium, provided the original work, first published in JMIR Research Protocols, is properly cited. The complete bibliographic information, a link to the original publication on http://www.researchprotocols.org, as well as this copyright and license information must be included. 